\title{
Language in psychiatry: a bedevilling dictionary
}

CLINICAL REFLECTION

\author{
Joseph M. Pierre \& Allan Frances
}

\begin{abstract}
SUMMARY
The language of psychiatry can be ambiguous and idiosyncratic, reflecting the elastic borders of mental illness and psychiatric disorder. This problem is not unique to psychiatry, but as the medical specialty moves closer towards a 'spectrum view' of mental illness, psychiatric terminology increasingly risks misappropriation and conflation with lay concepts of normal suffering. Deciding what words mean and how psychiatric disorders are defined requires ongoing consideration of the pragmatic consequences, both intended and unintended. Refining the lexicon of psychiatry with an eye towards precision and the minimisation of stigma requires that terms be revised and updated from time to time, but often suitable word replacements remain elusive.
\end{abstract}

\section{DECLARATION OF INTEREST}

None

\begin{abstract}
'When I use a word,'Humpty Dumpty said in rather a scornful tone, 'it means just what I choose it to mean - neither more nor less.'

'The question is,' said Alice, 'whether you can make words mean so many different things.'

'The question is,' said Humpty Dumpty, 'which is to be master - that's all.'
\end{abstract}

(Lewis Carroll, Through the Looking Glass, 1871)

You may recall that Humpty's complacent confidence in the power and precision of his words immediately preceded his very great fall - and that all the king's horses and all the king's men couldn't repair the damage done. The lesson for us is that if any psychiatric term can possibly be misused, it almost certainly will be.

Humpty's fundamental error was believing that it is possible to remain the master of our words and to control their connotations for, and usage by, others. Words tend to take on a life of their own, often becoming ambiguous and misleading in the process. Once an incorrect usage acquires the tenure of long and wide acceptance, it can be very difficult to control or replace.

The language of psychiatry is challenged by ambiguity, elasticity and idiosyncrasy in definitions and word usage (Frances 2010). These challenges are also present across all of medicine. As a construct, 'pathology' is fluid, and shifting demarcations of the normal/disease boundary result in strange hybrids such as pre-hypertension, pre-diabetes, predementia, carcinoma in situ, attenuated psychosis and minor depression. And there is often no clear boundary separating disease from the expectable wear and tear of life - 'normal', healthy people endure inevitable morbidity in the form of ageing, emotional suffering and physical pain, not to mention the certainty of death. Accordingly, there is no perfect operational definition of the most basic and commonplace medical terms such as disease, illness and health.

One of the most criticised and unsettling aspects of psychiatry is its inevitable inability to firmly define the borders of mental disorder (Frances 2010; Pierre 2010; Philips 2012). This has led some to the extreme and unreasonable conclusion that mental illness does not exist at all. Like any medical nosology, psychiatric diagnosis is on its firmest ground at the most severe end of the healthillness continuum and at its fuzziest in the grey areas between the normal and the pathological.

Diagnostic reliability suffers most with conditions and symptoms that are modelled along a spectrum and when there is confusion with normality and lay concepts. Psychiatric disorders should be understood as syndromes - that is, constellations of associated symptoms - not just hypertrophied traits. Clinicians must therefore take care not to lump dysphoria and everyday sadness together with major depression, normal 'ups and downs' with hypomania, shyness with social anxiety disorder, realistic worry with generalised anxiety disorder, or youthful distraction with attention-deficit hyperactivity disorder, or to conflate potentially adaptive psychological traits such as narcissism and obsessionality with personality disorder. Although insurance reimbursement and pharmaceutical marketing often incentivise doctors to do so, erring on the side of diagnosing a full-blown mental disorder risks overprescribing, unnecessary sideeffects, high placebo-response rates and inadequate use of non-pharmaceutical interventions such as self-help and psychotherapy. All this said, the interrater reliability of psychiatric disorders, while
Joseph M. Pierre is the Associate Director of Residency Education at UCLA Semel Institute for Neuroscience and the West LoS Angeles VA Medical Center, the Co-Chief of the Schizophrenia Treatment Unit, West Los Angeles VA Medical Center, and a Health Sciences Clinical Professor at the David Geffen School of Medicine at UCLA. Allan Frances is a Professor Emeritus at Duke University School of Medicine, the former Chair of the DSM-IV Task Force and the former Chair of the Department of Psychiatry at Duke University School of Medicine.

Correspondence $\operatorname{Dr}$ Joseph M. Pierre, Schizophrenia Treatment Unit, West Los Angeles VA Medical Center, 11301 Wilshere Boulevard, Bulding 210, Los Angeles, CA 90073, USA. Email: joseph.pierre2@va.gov 
less than desirable, is on a par with many other medical diagnoses, such as stroke, colon cancer and osteoarthritis (Pies 2007).

The elastic borders of mental illness require that clinicians act as fallible umpires, calling balls and strikes as best they see them (Frances 2010; Philips 2012). By necessity, the selection and use of diagnostic terms depend on contextual utility as defined by pragmatic considerations specific to the variety of situations in which diagnosis is used (Pierre 2010). We must think about the consequences, both intended and unintended, of definitions and word usage. So much rides on how we define words in psychiatry: who gets treated and how; who pays for it; whether a criminal is deemed mad or bad; whether someone gets damages in tort cases; who qualifies for disability payments and eligibility for extra school services; and whether someone can adopt a child. Ultimately, the most important consideration is whether a term will do more harm or good.

The National Institute of Mental Health proposed a whole new lexicon of research domain criteria, simplifying psychiatric terminology with the intention of making translational research easier. This new vocabulary remains largely untested in terms of reliability, validity and utility (Frances 2014). Although the endeavour may eventually improve the language of psychiatry, it is premature to throw out the language of the DSM. The DSM is nothing like the bible that many claim it to be, but it remains a 'good enough guide for clinical work' (Philips 2012).

\section{A bedevilling dictionary}

Paradoxically, some of the most commonly used terms in psychiatry are also its most misleading. The rub is that they also seem impossible to replace because their potential substitutes are even worse.

\section{Mental illness}

'Mental illness' is a misleading term, because the mental disorders we diagnose are no more than descriptions of what clinicians observe people doing or saying, rather than well-established diseases. The term lends itself to a simple-minded biological reductionism that pays insufficient attention to the psychological and social factors that are crucial in understanding anyone's problems. There is also no definition of mental illness that clearly distinguishes it from the equally undefinable term 'mental health'. Everyone complains about the term 'mental illness', but nobody has come up with a better substitute.

'Severely mentally ill' or its alternative, 'seriously mentally ill', are not only awkward tongue-twisters, but also misleading and potentially stigmatising.
They may be taken to mean that the person so labelled is destined to have a bad outcome and/ or that medication will be not only necessary in the treatment, but also sufficient. However, these terms also have no easy replacement and one or the other is necessary, because without some clearly recognisable designation this already shamefully neglected group would be even more ignored.

\section{Patient}

'Patient' has come to imply participation in a hierarchical relationship that brings with it little responsibility for shared decision-making. It is a mistake when doctors make unilateral decisions for their patients, because people's needs, tastes and wishes vary so much and there is usually such uncertainty about which treatments from a menu of plausible options might best suit that particular person. But substitute terms such as client, consumer, customer or service user are too cold, market-sounding and businesslike - lacking the connotations of caring and responsibility associated with helping a patient. And surveys show that many patients actually prefer to be called patients. Unless someone comes up with a better term, it is probably better to rehabilitate the connotation of patient rather than replace it, making it clear that it implies full partnership in a therapeutic relationship.

\section{Borderline personality disorder}

It is not at all clear what 'borderline personality disorder' is supposed to border on. Its original usage, now mostly archaic, derived from psychoanalysis and located it on the border between neurotic and psychotic levels of intrapsychic organisation. The term was later, confusingly, appropriated to imply a syndrome on the border of either mood or impulse disorder. Now the term has become a wastebasket diagnosis for a heterogeneous group of patients who have problems with interpersonal relationships, identity, impulse control, mood modulation control and self-destructive behaviour. How awkward to tell someone unsure of their personal identity that they are borderline - especially when there is no agreement regarding what they are bordering on. The best potential replacement term, 'emotionally unstable personality disorder', was considered for DSM-IV (1994), but (perhaps regrettably) rejected because borderline personality disorder was already so entrenched in the literature.

\section{Major depressive disorder}

The biggest mistake in DSM-III (1980) was introducing the very broad and heterogeneous term 'major depressive disorder', which combined what had previously been two separate and quite 
different presentations: (a) depression that is severe, delusional or incapacitating, and (b) depression that is mild, reactive to stress and often transient. The result is that many people get labelled as having major depressive disorder, even though their presentation isn't really major, depressive or a disorder. Mild sadness in reaction to stress and disappointment was lumped together with the most severe suffering known to man. Major depressive disorder has derived a tenure it doesn't deserve because it is now so ingrained in clinical and research usage. 'Melancholia' is a more specific, homogeneous term with a long pedigree that is embedded in the DSM as a severe subtype of depression, but is now too rarely used. Melancholia would provide many advantages if used more often in research, treatment planning and clinical communication (Parker 2010).

\section{Schizophrenia}

'Schizophrenia' is a problematic term that lacks lexical precision (just what is a 'split mind'?) and bears the inaccurate and stigmatising connotation of deterioration (many do recover). Schizophrenia is a broad, umbrella diagnosis that describes a heterogeneous set of experiences and behaviours with hundreds of potential causes and dozens of different treatments, rather than any one illness or unitary pathophysiological explanation. It has historically been used with harmful elasticity to inappropriately label milder or more transient conditions on the psychosis continuum. The Japanese have replaced schizophrenia with a more descriptive term that translates to 'integration disorder'. And yet, just as with melancholia, retaining the term schizophrenia to describe the more severe end of the psychosis spectrum could help to avoid confusion with experiences that are fundamentally different, such as non-pathological voice-hearing.

\section{Sticks and stones}

Labels can help a great deal. They can also hurt a great deal. They can provide clarity or badly mislead. The words we use to describe mental health and ill health carry heavy baggage of misleading and potentially stigmatising connotations. They are vastly overused to describe mild problems of everyday life better described with everyday language. Still, we need diagnostic terms, and most of the suggested replacements seem more harmful than helpful.

In the value-laden language of disease, where health is 'good' and illness is 'bad', stigma will always remain a concern. A person with heart disease might say 'I've got a bad ticker'. But we're loath to accept labels of mental illness because psychiatric disorders affect our brains and our behaviour, where our very identity is rooted.

Ultimately, though, stigma isn't caused by a name and isn't remedied by replacing it with a euphemism. It's caused by the way that other people regard someone affected by disease or disorder and the limitations of the ability of physicians to help. So, although we have done well to move beyond labels like 'cretin' or 'inadequate personality disorder', improving the treatment of mental illness and removing its association with poor functioning and poor prospects of recovery will go much further towards combating stigma than changing language ever will. The best way to reduce the stigma of mental illness is to treat the mentally ill well, with adequate services and housing, and to include them as participants in the social life of our communities.

\section{References}

American Psychiatric Association (1980) Diagnostic and Statistical Manual of Mental Disorders (3rd edn). APA.

American Psychiatric Association (1994) Diagnostic and Statistical Manual of Mental Disorders (4th edn). APA.

Frances A (2010) DSM in philosophyland: curiouser and curiouser. Association for the Advancement of Philosophy and Psychiatry (AAPP) Bulletin, 17: 3-7.

Frances A (2014) RDoC is necessary, but very oversold. World Psychiatry, 13: $47-9$.

Parker G, Fink M, Shorter E, et al (2010) Issues for DSM-5: whither melancholia? The case for its classification as a distinct mood disorder. American Journal of Psychiatry, 167: 745-7.

Philips J, Frances A, Cerullo MA, et al (2012) The six most essential questions in psychiatric diagnosis: a pluralogue part 1: conceptual and definitional issues in psychiatric diagnosis. Philosophy, Ethics, and Humanities in Medicine, 70: 3

Pierre JM (2010) The borders of mental disorder in psychiatry and the DSM: past, present, future. Journal of Psychiatric Practice, 16: 375-86.

Pies R (2007) How 'objective' are psychiatric diagnoses? (Guess again) Current Psychiatry, 6: 18-22. 